\title{
A prime analogue of the Erdös-Pomerance conjecture for elliptic curves
}

Yu-Ru Liu*

Abstract. Let $E / \mathbb{Q}$ be an elliptic curve of rank $\geq 1$ and $b \in E(\mathbb{Q})$ a rational point of infinite order. For a prime $p$ of good reduction, let $g_{b}(p)$ be the order of the cyclic group generated by the reduction $\bar{b}$ of $b$ modulo $p$. We denote by $\omega\left(g_{b}(p)\right)$ the number of distinct prime divisors of $g_{b}(p)$. Assuming the GRH, we show that the normal order of $\omega\left(g_{b}(p)\right)$ is $\log \log p$. We also prove conditionally that there exists a normal distribution for the quantity

$$
\frac{\omega\left(g_{b}(p)\right)-\log \log p}{\sqrt{\log \log p}} .
$$

The latter result can be viewed as an elliptic analogue of a conjecture of Erdös and Pomerance about the distribution of $\omega\left(f_{a}(n)\right)$, where $a$ is a natural number $>1$ and $f_{a}(n)$ the order of $a$ modulo $n$.

Mathematics Subject Classification (2000). 11N37, 11G20.

Keywords. Prime divisors, order of cyclic groups, elliptic curves.

\section{Introduction}

For $n \in \mathbb{N}:=\{1,2,3, \ldots\}$, let $\omega(n)$ denote the number of distinct prime divisors of $n$. The Turán Theorem is about the second moment of $\omega(n)$ [23]; it states that for $x \in \mathbb{R}, x>1$,

$$
\sum_{n \leq x}(\omega(n)-\log \log x)^{2} \ll x \log \log x .
$$

Turán's result implies an earlier theorem of Hardy and Ramanujan [8], which states that for any $\varepsilon>0$

$$
\#\{n \leq x \mid n \text { satisfies }|\omega(n)-\log \log n|>\varepsilon \log \log n\}
$$

is $o(x)$ as $x \rightarrow \infty$. In other words, the normal order of $\omega(n)$ is $\log \log n$. The significance of the ' $\log \log n$ ' term is that it is about $\sum_{p \leq n} \frac{\omega(p)}{p}$ where $p$ runs over primes.

\footnotetext{
${ }^{*}$ Research partially supported by an NSERC discovery grant.
} 
The idea behind Turán's proof was essentially probabilistic. Further development of probabilistic ideas led Erdös and Kac [5] to prove a remarkable refinement of the Turán Theorem, namely, the existence of a normal distribution for $\omega(n)$. More precisely, they proved that for $\gamma \in \mathbb{R}$,

$\lim _{x \rightarrow \infty} \frac{1}{x} \#\left\{n \leq x \mid n\right.$ satisfies $\left.\frac{\omega(n)-\log \log n}{\sqrt{\log \log n}} \leq \gamma\right\}=G(\gamma):=\frac{1}{\sqrt{2 \pi}} \int_{-\infty}^{\gamma} e^{\frac{-t^{2}}{2}} d t$.

The theorem of Erdös and Kac opened a door to the study of probabilistic number theory. In the early 1960s and subsequently the 1970s, the theory was refined by many authors, culminating in a generalized Erdös-Kac theorem proved independently by Kubilius [10] and Shapiro [20]. Their result is applicable to what are called 'strongly additive functions'. The interested reader can find a comprehensive treatment of it in the monograph of Elliott [3].

We can also consider functions that are not strongly additive, say the Euler's $\varphi$-function. Using the same principle of the work of Kubilius and Shapiro, the issue of $\omega(\varphi(n))$ devolves upon the estimation of the sums

$$
\sum_{p \leq x} \omega(p-1) \text { and } \sum_{p \leq x} \omega^{2}(p-1)
$$

where $p$ denotes a rational prime. Sums of this type were estimated by Haselgrove [9] and Erdös and Pomerance [6]. They proved that

$$
\sum_{p \leq x} \omega(p-1)=\pi(x) \log \log x+O(\pi(x))
$$

and

$$
\sum_{p \leq x} \omega^{2}(p-1)=\pi(x)(\log \log x)^{2}+O(\pi(x) \log \log x),
$$

where $\pi(x)$ is the number of rational primes $\leq x$. Applying partial summation, we can derive from the above equalities that

$$
\sum_{p \leq n} \frac{\omega(p-1)}{p}=\frac{1}{2}(\log \log n)^{2}+O(\log \log n)
$$

and

$$
\sum_{p \leq n} \frac{\omega^{2}(p-1)}{p}=\frac{1}{3}(\log \log n)^{3}+O\left((\log \log n)^{2}\right) .
$$

As a consequence we have the following result of Erdös and Pomerance [6], which states that

$$
\lim _{x \rightarrow \infty} \frac{1}{x} \#\left\{n \leq x \mid n \text { satisfies } \frac{\omega(\varphi(n))-\frac{1}{2}(\log \log n)^{2}}{\frac{1}{\sqrt{3}}(\log \log n)^{3 / 2}} \leq \gamma\right\}=G(\gamma) .
$$


Vol. 80 (2005) A prime analogue of the Erdös-Pomerance conjecture for elliptic curves 757

In [6], Erdös and Pomerance also proposed the following question. Let $a$ be a positive integer $>1$. For any natural number $n$ coprime to $a$, let $f_{a}(n)$ denote the order of $a$ modulo $n$. Thus $f_{a}(n)$ is a divisor of $\varphi(n)$. Based on the belief that the difference between $\omega(\varphi(n))$ and $\omega\left(f_{a}(n)\right)$ is 'small on average', Erdös and Pomerance conjectured that

$$
\begin{aligned}
\lim _{x \rightarrow \infty} \frac{1}{x} \#\left\{n \leq x \mid n \text { satisfies }(a, n)=1 \text { and } \frac{\omega\left(f_{a}(n)\right)-\frac{1}{2}(\log \log n)^{2}}{\frac{1}{\sqrt{3}}(\log \log n)^{3 / 2}} \leq \gamma\right\} \\
=\frac{\varphi(a)}{a} G(\gamma) .
\end{aligned}
$$

The conjecture remains open until today. Even a conditional result was only obtained recently by Murty and Saidak [17] under the assumption of the GRH (i.e., the Riemann Hypothesis for all Dedekind zeta functions of number fields). Later Li and Pomerance [13] also provided an alternative proof of the same result. The difficulty of this conjecture lies in the intervention of the distribution of primes in the non-abelian extensions $\mathbb{Q}\left(\zeta_{q}, \sqrt[q]{a}\right)$ where $q$ varies over rational primes and $\zeta_{q}$ is a primitive $q$-th root of unity.

Let us recall that $f_{a}(n)$ is the least common multiple of $\left\{f_{a}\left(p^{\gamma}\right) \mid p^{\gamma} \| n\right\}$ where $p^{\gamma}$ is the exact power of $p$ which divides $n$. Also $f_{a}\left(p^{\gamma}\right)$ divides $p^{\gamma-1} f_{a}(p)$. Thus similarly to the case of $\omega(\varphi(n))$, to study the conjecture of Erdös and Pomerance, it is sufficient to estimate the sums

$$
\sum_{p \leq x} \omega\left(f_{a}(p)\right) \text { and } \sum_{p \leq x} \omega^{2}\left(f_{a}(p)\right)
$$

Under the assumption of the GRH, Murty and Saidak proved that

$$
\sum_{p \leq x} \omega\left(f_{a}(p)\right)=\pi(x) \log \log x+O(\pi(x))
$$

and

$$
\sum_{p \leq x} \omega^{2}\left(f_{a}(p)\right)=\pi(x)(\log \log x)^{2}+O(\pi(x) \log \log x) .
$$

A conditional result of the conjecture follows.

In [17], Murty and Saidak also proved the following 'prime analogue' of the Erdös-Pomerance conjecture:

$$
\begin{aligned}
\lim _{x \rightarrow \infty} \frac{1}{\pi(x)} & \#\left\{p \leq x \mid p \text { satisfies }(a, p)=1 \text { and } \frac{\omega\left(f_{a}(p)\right)-\log \log p}{\sqrt{\log \log p}} \leq \gamma\right\} \\
= & G(\gamma) .
\end{aligned}
$$


In a sense, as we see from $[17, \S 5, \S 7]$, there is not much difference between the study of $\omega\left(f_{a}(n)\right)$ and $\omega\left(f_{a}(p)\right)$, as the main technical difficulty of both problems depends on the study of $\omega\left(i_{a}(p)\right)$, where $i_{a}(p)=(p-1) / f_{a}(p)$.

The purpose of this paper is to formulate an analogous Erdös-Pomerance conjecture for elliptic curves and provide a conditional proof of it. Let $E / \mathbb{Q}$ be an elliptic curve of rank $\geq 1$. Let $b \in E(\mathbb{Q})$ be a rational point of infinite order. For a prime $p$ of good reduction, let $g_{b}(p)$ be the order of $\langle\bar{b}\rangle$, the cyclic group generated by the reduction $\bar{b}$ of $b$ modulo $p$. The function $g_{b}(p)$ can be viewed as an elliptic analogue of $f_{a}(p)$. Thus, an analogous formulation of the conjecture of Erdös and Pomerance for elliptic curves is that there exists a normal distribution for the quantity

$$
\frac{\omega\left(g_{b}(p)\right)-\log \log p}{\sqrt{\log \log p}} .
$$

We prove the following result.

Theorem 1. Let $E / \mathbb{Q}$ be an elliptic curve of rank $\geq 1$ and $b \in E(\mathbb{Q})$ a rational point of infinite order. For a prime $p$ of good reduction, let $\langle\bar{b}\rangle$ be the cyclic group generated by the reduction $\bar{b}$ of $b$ modulo $p$ and $g_{b}(p)$ its order. Assuming the GRH, we have

$$
\sum_{\substack{p \leq x \\ p \text { of good reduction }}}\left(\omega\left(g_{b}(p)\right)-\log \log x\right)^{2} \ll \pi(x) \log \log x .
$$

As a direct consequence of Theorem 1 we have

Corollary 2. Assuming the GRH, the normal order of $\omega\left(g_{b}(p)\right)$ is $\log \log p$.

The following theorem is an analogous result of Murty and Saidak for elliptic curves.

Theorem 3. Let $E / \mathbb{Q}, b$, and $g_{b}(p)$ be defined as in Theorem 1 . Let $\gamma \in \mathbb{R}$. Assuming the GRH, we have

$$
\begin{aligned}
& \lim _{x \rightarrow \infty} \frac{1}{\pi(x)} \#\left\{p \leq x \mid p \text { is of good reduction and } \frac{\omega\left(g_{b}(p)\right)-\log \log p}{\sqrt{\log \log p}} \leq \gamma\right\} \\
& =G(\gamma) .
\end{aligned}
$$

Thus, we obtain an elliptic analogue of a conjecture of Erdös and Pomerance in terms of primes.

Acknowledgment. I would like to thank W. Kuo and R. Murty for many helpful discussions related to this work. I also would like to thank D. Mckinnon for his 
Vol. 80 (2005) A prime analogue of the Erdös-Pomerance conjecture for elliptic curves 759

comments about this paper. Special thanks go to the referee for the careful reading of the paper and many valuable suggestions.

Notation. For $x \in \mathbb{R}, x>0$, let $f(x)$ and $g(x)$ be two functions of $x$. If $g(x)$ is positive and there exists a constant $C>0$ such that $|f(x)| \leq C g(x)$, we write either $f(x) \ll g(x)$ or $f(x)=O(g(x))$. If both $f(x)$ and $g(x)$ are positive, we use $f(x) \asymp g(x)$ to denote that $f(x)=O(g(x))$ and $g(x)=O(f(x))$. If

$\lim _{x \rightarrow \infty} \frac{f(x)}{g(x)}=0$, we write $f(x)=o(g(x))$. Also, we use $\overline{\mathbb{Q}}$ and $\overline{\mathbb{F}}_{p}$ to denote some fixed algebraic closures of $\mathbb{Q}$ and $\mathbb{F}_{p}$ respectively.

\section{Preliminaries}

We first recall some theorems about elliptic curves that will be needed later. Let $E / \mathbb{Q}$ be an elliptic curve of rank $\geq 1$. For a prime $l \in \mathbb{N}$, we denote by $E[l]$ the $l$-torsion points. By adjoining to $\mathbb{Q}$ the coordinates of the $l$-torsion points, we obtain $\mathbb{Q}(E[l])$, a finite Galois extension of $\mathbb{Q}$. Since

$$
E[l] \cong(\mathbb{Z} / l \mathbb{Z}) \times(\mathbb{Z} / l \mathbb{Z})
$$

(see [21, Corollary 6.4]), by choosing a basis, we have a natural injection

$$
\Phi_{l}: \operatorname{Gal}(\mathbb{Q}(E[l]) / \mathbb{Q}) \hookrightarrow \mathrm{GL}_{2}(\mathbb{Z} / l \mathbb{Z}) .
$$

In the following discussion we will abuse our notation by identifying an element $\gamma \in \operatorname{Gal}(\mathbb{Q}(E[l]) / \mathbb{Q})$ with its image $\Phi_{l}(\gamma) \in \mathrm{GL}_{2}(\mathbb{Z} / l \mathbb{Z})$.

Let $b \in E(\mathbb{Q})$ be a rational point of infinite order. We denote by $l^{-1} b$ the set of elements $v \in E(\overline{\mathbb{Q}})$ such that

$$
[l] v=\underbrace{v+v+\cdots+v}_{l \text { times }}=b .
$$

Define $L_{l}=\mathbb{Q}\left(E[l], l^{-1} b\right)$, which is a finite extension of $\mathbb{Q}(E[l])$. We have the following theorem.

Theorem 4 (Bachmakov [1]). For a prime l, the Galois group $\operatorname{Gal}\left(L_{l} / \mathbb{Q}(E[l])\right)$ can be identified with a subgroup of $E[l]$ and is equal to $E[l]$ for all but finitely many $l$.

The group $\mathrm{GL}_{2}(\mathbb{Z} / l \mathbb{Z})$ acts naturally on $E[l]$ by matrix multiplication. We denote this action by $*$ and we see that it induces a semidirect product $E[l] \rtimes \mathrm{GL}_{2}(\mathbb{Z} / l \mathbb{Z})$. Let $G_{l}$ be the Galois group $\operatorname{Gal}\left(L_{l} / \mathbb{Q}\right)$. From Theorem 4 , for all but finitely many $l$, we have

$$
G_{l} \cong E[l] \rtimes \operatorname{Gal}(\mathbb{Q}(E[l]) / \mathbb{Q}),
$$

which is a subgroup of $E[l] \rtimes \mathrm{GL}_{2}(\mathbb{Z} / l \mathbb{Z})$. 
An element $(\tau, \gamma) \in G_{l}$ acts on $E[l]$ and $l^{-1} b$ as follows: let $v_{0} \in l^{-1} b$ be a fixed element; for $u \in E[l]$ and $v \in l^{-1} b$ we have

- $(\tau, \gamma) \cdot u:=\gamma * u$.

- $(\tau, \gamma) \cdot v:=v_{0}+\gamma *\left(v-v_{0}\right)+\tau$.

Notice that since $[l] v=[l] v_{0}=b,\left(v-v_{0}\right) \in E[l]$. Thus, $\gamma *\left(v-v_{0}\right)$ is well defined. Also, since both $\left(v-v_{0}\right)$ and $\tau$ are in $E[l]$, for $v \in l^{-1} b$, we have

$$
[l]((\tau, \gamma) \cdot v)=[l] v_{0}=b .
$$

Thus, $(\tau, \gamma)$ is a well-defined action on the set $l^{-1} b$. Moreover, for $v \in l^{-1} b$, we have

$$
(\tau, \gamma) \cdot v=v \quad \text { if and only if } \quad(\gamma-I) *\left(v_{0}-v\right)=\tau,
$$

where $I$ is the $2 \times 2$ identity matrix.

Let $p$ be a prime of good reduction. We denote by $\bar{E}$ the reduction of $E$ modulo $p$. Let $\bar{E}\left(\mathbb{F}_{p}\right)$ be the set of rational points of $\bar{E}$ defined over the finite field $\mathbb{F}_{p}$. Let $b \in E(\mathbb{Q})$ be a rational point of infinite order and $\bar{b} \in \bar{E}\left(\mathbb{F}_{p}\right)$ the reduction of $b$ modulo $p$. Let $\langle\bar{b}\rangle$ be the cyclic group generated by $\bar{b}$, which is a subgroup of $\bar{E}\left(\mathbb{F}_{p}\right)$. We denote by $g_{b}(p)$ the order of $\langle\bar{b}\rangle$. Thus $g_{b}(p)$ is a divisor of $\# \bar{E}\left(\mathbb{F}_{p}\right)$. We write

$$
\# \bar{E}\left(\mathbb{F}_{p}\right)=g_{b}(p) \cdot i_{b}(p),
$$

where $i_{b}(p)$ is the index of $\langle\bar{b}\rangle$ in $\bar{E}\left(\mathbb{F}_{p}\right)$. Let $\Delta$ be the discriminant of $E$. For $p \nmid l \Delta$, Lang and Trotter [12] gave a condition on the Frobenius element $\left(\tau_{p}, \gamma_{p}\right) \in G_{l}$ in order that $l \mid i_{b}(p)$. We review their arguments below.

Notice that $l \mid i_{b}(p)$ implies that $l \mid \# \bar{E}\left(\mathbb{F}_{p}\right)$. Since

$$
\operatorname{tr} \gamma_{p} \equiv p+1-\# \bar{E}\left(\mathbb{F}_{p}\right)(\bmod l)
$$

and

$$
\operatorname{det} \gamma_{p} \equiv p(\bmod l)
$$

(see [22, p. 172]), if $l \mid \# \bar{E}\left(\mathbb{F}_{p}\right)$, we have

$$
1-\operatorname{tr} \gamma_{p}+\operatorname{det} \gamma_{p} \equiv 0(\bmod l) .
$$

Thus $\gamma_{p} \in \operatorname{Gal}(\mathbb{Q}(E[l]) / \mathbb{Q}) \subseteq \mathrm{GL}_{2}(\mathbb{Z} / l \mathbb{Z})$ has an eigenvalue 1 .

We consider first the case when $\gamma_{p}=I$. We recall that the cyclic group generated by $\pi_{p}: x \mapsto x^{p}$ is dense in $\operatorname{Gal}\left(\overline{\mathbb{F}}_{p} / \mathbb{F}_{p}\right)$. The group $\operatorname{Gal}\left(\overline{\mathbb{F}}_{p} / \mathbb{F}_{p}\right)$ acts on $w \in \bar{E}\left(\overline{\mathbb{F}}_{p}\right)$ coordinatewise. Thus for $w \in \bar{E}\left(\overline{\mathbb{F}}_{p}\right)$ we have

$$
\pi_{p} \cdot w=w \text { if and only if } \quad w \in \bar{E}\left(\mathbb{F}_{p}\right) .
$$


Vol. 80 (2005) A prime analogue of the Erdös-Pomerance conjecture for elliptic curves 761

Let $w_{1} \in E(\mathbb{Q}(E[l]))$. The Frobenius element $\gamma_{p} \in \operatorname{Gal}(\mathbb{Q}(E[l]) / \mathbb{Q})$ acts on $w_{1}$ coordinatewise. This action is compatible with $\pi_{p}$ in the following sense: let $\bar{w}_{1} \in$ $\bar{E}\left(\overline{\mathbb{F}}_{p}\right)$ be the reduction of $w_{1}$ modulo $p$; we have

$$
\overline{\gamma_{p} \cdot w_{1}}=\pi_{p} \cdot \bar{w}_{1} .
$$

Thus for $\gamma_{p}=I$ we have

$$
\bar{w}_{1}=\overline{\gamma_{p} \cdot w_{1}}=\pi_{p} \cdot \bar{w}_{1} .
$$

It follows that $\bar{w}_{1} \in \bar{E}\left(\mathbb{F}_{p}\right)$. Let $\bar{E}[l]$ denote the reduction of $E[l]$ modulo $p$. Since $E[l] \subseteq E(\mathbb{Q}(E[l]))$, the above argument shows that

$$
\bar{E}\left(\mathbb{F}_{p}\right) \supseteq \bar{E}[l] \cong(\mathbb{Z} / l \mathbb{Z}) \times(\mathbb{Z} / l \mathbb{Z}), \quad \text { provided that } p \nmid l \Delta
$$

(see [21, Corollary 6.4]). Consider the subgroup $\langle\bar{b}\rangle$ in $\bar{E}\left(\mathbb{F}_{p}\right)$. Since $\langle\bar{b}\rangle$ is cyclic, it can not contain two $(\mathbb{Z} / l \mathbb{Z})$ factors. Thus, at least one of $(\mathbb{Z} / l \mathbb{Z})$ factors of $\bar{E}\left(\mathbb{F}_{p}\right)$ is contained in $\bar{E}\left(\mathbb{F}_{p}\right) /\langle\bar{b}\rangle$. Since $i_{b}(p)$ is the order of $\bar{E}\left(\mathbb{F}_{p}\right) /\langle\bar{b}\rangle$, we have $l \mid i_{b}(p)$. We conclude that for $\gamma_{p}=I, l$ is a divisor of $i_{b}(p)$.

On the other hand, if $\gamma_{p}$ has an eigenvalue 1 and $\gamma_{p} \neq I, \bar{E}\left(\mathbb{F}_{p}\right)$ can not contain a $(\mathbb{Z} / l \mathbb{Z}) \times(\mathbb{Z} / l \mathbb{Z})$ factor. Hence, the $l$-torsion points of $\bar{E}\left(\mathbb{F}_{p}\right)$, which is the kernel of the map $\gamma_{p}-I: E[l] \rightarrow E[l]$, form a cyclic subgroup. In other words, the $l$-primary part of $\bar{E}\left(\mathbb{F}_{p}\right)$ is of the form $\mathbb{Z} / l^{\alpha} \mathbb{Z}$ for some $\alpha \in \mathbb{N}$. Write

$$
\bar{E}\left(\mathbb{F}_{p}\right) \cong \mathbb{Z} / l^{\alpha} \mathbb{Z} \times H,
$$

where $H$ is an abelian group with $(|H|, l)=1$. We will abuse our notation by identifying an element in $\bar{E}\left(\mathbb{F}_{p}\right)$ with its image in $\mathbb{Z} / l^{\alpha} \mathbb{Z} \times H$. For $\bar{b} \in \bar{E}\left(\mathbb{F}_{p}\right)$, without loss of generality, we can assume that either $\bar{b}=(0, h)$ or $\bar{b}=\left(l^{\beta}, h\right)$ where $h \in H$ and $\beta \geq 0$.

Case 1. Suppose $\bar{b}=(0, h)$. Since $(|H|, l)=1$, the element $\bar{b}_{l}=\left(0, l^{-1} h\right) \in$ $\bar{E}\left(\mathbb{F}_{p}\right)$ is well defined and $[l] \bar{b}_{l}=\bar{b}$.

Case 2. Suppose $\bar{b}=\left(l^{\beta}, h\right)$. If $\beta=0$, the order of the cyclic group $\langle b\rangle$ is divisible by $l^{\alpha}$, i.e., $l \nmid i_{b}(p)$. Hence, if $l \mid i_{b}(p)$, it implies that $\beta \geq 1$. Choosing $\bar{b}_{l}=\left(l^{\beta-1}, l^{-1} h\right) \in \bar{E}\left(\mathbb{F}_{p}\right)$, we have $[l] \bar{b}_{l}=\bar{b}$.

We conclude that if $\gamma_{p}$ has an eigenvalue $1, \gamma_{p} \neq 1$ and $l \mid i_{b}(p)$, there exists $\bar{b}_{l} \in \bar{E}\left(\mathbb{F}_{p}\right)$ such that $[l] \bar{b}_{l}=\bar{b}$. Let $b_{l} \in \bar{E}(\overline{\mathbb{Q}})$ such that the reduction of $b_{l}$ modulo $p$ is $\bar{b}_{l}$. Since $[l] \bar{b}_{l}=\bar{b}$, it follows that $b_{l} \in l^{-1} b$. Moreover, since $\bar{b}_{l} \in E\left(\mathbb{F}_{p}\right)$, we have

$$
\left(\tau_{p}, \gamma_{p}\right) \cdot b_{l}=b_{l},
$$


which is equivalent to

$$
\left(\gamma_{p}-I\right) *\left(v_{0}-b_{l}\right)=\tau_{p}
$$

i.e., $\tau_{p} \in \operatorname{Im}\left(\gamma_{p}-I\right)$.

Define a subset $S_{l}$ of $G_{l}$ as follows: an element $(\tau, \gamma)$ of $G_{l}$ belongs to $S_{l}$ if it satisfies one of the two following conditions:

(1) $\gamma=I$ or

(2) $\gamma$ has an eigenvalue $1, \operatorname{ker}((\gamma-I): E[l] \rightarrow E[l])$ is cyclic, and $\tau \in \operatorname{Im}(\gamma-I)$.

Notice that $S_{l}$ is a union of conjugacy classes of $G_{l}$. Combining all the above discussions, we obtain the following result of Lang and Trotter.

Theorem 5 (Lang and Trotter [12]). Let $i_{b}(p)$ be the index of the cyclic group $\langle\bar{b}\rangle$ in $\bar{E}\left(\mathbb{F}_{p}\right)$. For a prime $l \in \mathbb{N}, p \nmid l \Delta$, the following two statements are equivalent:

(1) $l \mid i_{b}(p)$.

(2) $\left(\tau_{p}, \gamma_{p}\right) \in S_{l}$.

Another important ingredient of the proof of Theorems 1 and 3 is the Chebotarev density theorem. Let $L / \mathbb{Q}$ be a finite Galois extension of degree $n_{L}$ and discriminant $d_{L}$. We denote by $G$ the Galois group of $L / \mathbb{Q}$ and $C$ a union of conjugacy classes of $G$. Let $\sigma_{p} \in G$ be a Frobenius element. Define

$$
\pi_{C}(x, L / \mathbb{Q})=\#\left\{p \leq x \mid p \text { is an unramified prime in } L / \mathbb{Q} \text { and } \sigma_{p} \subseteq C\right\} .
$$

We have

Theorem 6 (Lagarias and Odlyzko [11], Serre [19]). Assuming the GRH for the Dedekind zeta function of $L$, we have

$$
\pi_{C}(x, L / \mathbb{Q})=\frac{|C|}{|G|} \operatorname{li} x+O\left(|C| x^{\frac{1}{2}}\left(\frac{\log \left|d_{L}\right|}{n_{L}}+\log x\right)\right),
$$

where li $x=\int_{2}^{x} \frac{d t}{\log t}$.

The following theorem is useful for estimating the error term in the Chebotarev density theorem.

Theorem 7 (Serre [19]). Let $L / \mathbb{Q}$ be a finite Galois extension of degree $n_{L}$ and discriminant $d_{L}$. We have

$$
\frac{n_{L}}{2} \sum_{q \text { ramified }} \log q \leq \log \left|d_{L}\right| \leq\left(n_{L}-1\right) \sum_{q \text { ramified }} \log q+n_{L} \log n_{L},
$$

where the sum is over all primes $q$ that are ramified in $L$. 
Vol. 80 (2005) A prime analogue of the Erdös-Pomerance conjecture for elliptic curves 763

\section{Prime divisors of $i_{b}(p)$}

We recall that $i_{b}(p)$ is the index of $\langle\bar{b}\rangle$ in $\bar{E}\left(\mathbb{F}_{p}\right)$. In this section, we consider the number of distinct prime divisors of $i_{b}(p)$. The following lemma is essential for the proof of Theorems 1 and 3 . We use the notation $\sum^{\prime}$ to denote the sum over primes of good reduction.

Lemma 8. Assuming the GRH, we have

$$
\sum_{p \leq x}^{\prime} \omega^{2}\left(i_{b}(p)\right) \ll \pi(x)
$$

Proof. Let $y=x^{\delta}$ with $0<\delta<1$ (a choice of $\delta$ will be made later). Define a truncation function $\omega_{y}$ of $\omega$ as follows:

$$
\omega_{y}\left(i_{b}(p)\right)=\#\left\{l \leq y \mid l \text { is a prime and } l \mid i_{b}(p)\right\} .
$$

For a prime $p \leq x$, since

$$
i_{b}(p) \leq \# \bar{E}\left(\mathbb{F}_{p}\right) \leq(p+2 \sqrt{p}+1) \leq 3 x,
$$

it follows that

$$
\omega\left(i_{b}(p)\right)=\omega_{y}\left(i_{b}(p)\right)+O(1)
$$

Hence we have

$$
\begin{aligned}
\sum_{p \leq x}^{\prime} \omega^{2}\left(i_{b}(p)\right) & =\sum_{p \leq x}^{\prime}\left(\omega_{y}\left(i_{b}(p)\right)+O(1)\right)^{2} \ll \sum_{p \leq x}^{\prime} \omega_{y}^{2}\left(i_{b}(p)\right)+O(\pi(x)) \\
& =\sum_{\substack{l_{1}, l_{2} \leq y \\
l_{1} \neq l_{2}}} \sum_{\substack{p \leq x \\
l_{1} l_{2} \mid i_{b}(p)}}^{\prime} 1+\sum_{l \leq y} \sum_{\substack{p \leq x \\
l \mid i_{b}(p)}}^{\prime} 1+O(\pi(x)),
\end{aligned}
$$

where $l_{1}, l_{2}$, and $l$ are rational primes. Consider the sum

$$
\sum_{l \leq y} \sum_{\substack{p \leq x \\ l \mid i_{b}(p)}}^{\prime} 1 .
$$

Applying Theorems 5, 6 and 7 for all but finitely many primes $l$, under the GRH we have

$$
\begin{aligned}
\#\{p & \left.\leq x \mid p \text { satisfies } l \mid i_{b}(p)\right\} \\
& =\operatorname{li} x \cdot \frac{\left|S_{l}\right|}{\left|G_{l}\right|}+O\left(\left|S_{l}\right| \cdot x^{\frac{1}{2}} \cdot\left(\sum_{q \text { ramified }} \log q++\log n_{l}+\log x\right)\right),
\end{aligned}
$$

where the sum is over all primes $q$ that are ramified in $L_{l}$ and $n_{l}=\left|G_{l}\right|$. 
In the case of elliptic curves without complex multiplication (non-CM) Serre [18] proved that for all but finitely many primes $l$,

$$
\operatorname{Gal}(\mathbb{Q}(E[l]) / \mathbb{Q})=\mathrm{GL}_{2}(\mathbb{Z} / l \mathbb{Z}) .
$$

Hence, for all but finitely many $l$, we have

$$
\left|G_{l}\right| \asymp l^{6} \text { and }\left|S_{l}\right| \asymp l^{4} .
$$

In the case of elliptic curves with complex multiplication (CM), from [7, p. 35-37], we have

$$
\left|G_{l}\right| \asymp l^{4} \text { and }\left|S_{l}\right| \asymp l^{2} .
$$

It is well known that $q$ is ramified in $L_{l}$ if and only if $q \mid l \Delta$ (see [2]). Hence, assuming the GRH, we have

$$
\sum_{l \leq y} \sum_{\substack{p \leq x \\ l \mid i_{b}(p)}}^{\prime} 1 \ll \sum_{l \leq y}\left(\frac{\pi(x)}{l^{2}}+O\left(l^{4} x^{\frac{1}{2}} \log \left(l^{6} x \Delta\right)\right)\right) \ll \pi(x)+O\left(x^{\frac{1}{2}+5 \delta+\varepsilon}\right),
$$

where $\varepsilon>0$ is arbitrarily small. Choosing $\delta=\frac{1}{11}$, we have

$$
\sum_{l \leq y} \sum_{\substack{p \leq x \\ l \mid i_{b}(p)}}^{\prime} 1 \ll \pi(x)
$$

Consider the sum

$$
\sum_{\substack{l_{1}, l_{2} \leq y \\ l_{1} \neq l_{2}}} \sum_{\substack{p \leq x \\ l_{1} l_{2} \mid i_{b}(p)}}^{\prime} 1
$$

The group homomorphisms

$$
E\left[l_{1} l_{2}\right] \rightarrow E\left[l_{1}\right] \times E\left[l_{2}\right] \text { and } \mathrm{GL}_{2}\left(\mathbb{Z} / l_{1} l_{2} \mathbb{Z}\right) \rightarrow \mathrm{GL}_{2}\left(\mathbb{Z} / l_{1} \mathbb{Z}\right) \times \mathrm{GL}_{2}\left(\mathbb{Z} / l_{2} \mathbb{Z}\right) \text {, }
$$

which are induced by reduction modulo $l_{1}$ and $l_{2}$ respectively, are indeed isomorphisms. Moreover, these maps are compatible with the actions defined in Section 2. Since $\left|S_{l}\right| /\left|G_{l}\right| \asymp 1 / l^{2}$, by Theorems 5, 6 and 7 we have

$$
\begin{aligned}
\sum_{\substack{l_{1}, l_{2} \leq y \\
l_{1} \neq l_{2}}} \sum_{\substack{p \leq x \\
l_{1} l_{2} \mid i_{b}(p)}}^{\prime} 1 \ll \sum_{\substack{l_{1}, l_{2} \leq y \\
l_{1} \neq l_{2}}}\left(\frac{\pi(x)}{\left(l_{1} l_{2}\right)^{2}}+O\left(\left(l_{1} l_{2}\right)^{4} x^{\frac{1}{2}} \log \left(l_{1}^{6} l_{2}^{6} x \Delta\right)\right)\right) \\
\ll \pi(x)+O\left(x^{\frac{1}{2}+10 \delta+\varepsilon}\right),
\end{aligned}
$$

where $\varepsilon \rightarrow 0$ as $x \rightarrow \infty$. Choosing $\delta=\frac{1}{21}$, we have

$$
\sum_{\substack{l_{1}, l_{2} \leq y \\ l_{1} \neq l_{2}}} \sum_{\substack{p \leq x \\ l_{1} l_{2} \mid i_{b}(p)}}^{\prime} 1 \ll \pi(x)
$$


Vol. 80 (2005) A prime analogue of the Erdös-Pomerance conjecture for elliptic curves 765

It follows that

$$
\sum_{p \leq x}^{\prime} \omega^{2}\left(i_{b}(p)\right) \ll \pi(x) .
$$

This completes the proof of Lemma 8.

\section{A Turán analogue of $\omega\left(g_{b}(p)\right)$}

In this section, we provide a proof of Theorem 1 which states that under the GRH, we have

$$
\sum_{p \leq x}^{\prime}\left(\omega\left(g_{b}(p)\right)-\log \log x\right)^{2} \ll \pi(x) \log \log x .
$$

Our proof is a combination of Lemma 8 with the following theorem.

Theorem 9 (Miri and Murty [16], Liu [14]). Let $E / \mathbb{Q}$ be an elliptic curve. We have (assuming the GRH if $E$ is non-CM)

$$
\sum_{p \leq x}^{\prime}\left(\omega\left(\# \bar{E}\left(\mathbb{F}_{p}\right)\right)-\log \log x\right)^{2} \ll \pi(x) \log \log x .
$$

Now we are ready to prove Theorem 1.

Proof of Theorem 1. Since

$$
\# \bar{E}\left(\mathbb{F}_{p}\right)=g_{b}(p) \cdot i_{b}(p)
$$

we have

$$
\omega\left(\# \bar{E}\left(\mathbb{F}_{p}\right)\right) \geq \omega\left(g_{b}(p)\right) \geq \omega\left(\# \bar{E}\left(\mathbb{F}_{p}\right)\right)-\omega\left(i_{b}(p)\right) .
$$

It follows that

$$
\begin{aligned}
\sum_{p \leq x}^{\prime}\left(\omega\left(g_{b}(p)\right)-\log \log x\right)^{2} & =\sum_{p \leq x}^{\prime}\left(\omega\left(\# \bar{E}\left(\mathbb{F}_{p}\right)\right)+O\left(\omega\left(i_{b}(p)\right)\right)-\log \log x\right)^{2} \\
& \ll \sum_{p \leq x}^{\prime}\left(\omega\left(\# \bar{E}\left(\mathbb{F}_{p}\right)\right)-\log \log x\right)^{2}+\sum_{p \leq x}^{\prime} \omega^{2}\left(i_{b}(p)\right) .
\end{aligned}
$$

Combining Lemma 8 with Theorem 9 we obtain that under the GRH,

$$
\sum_{p \leq x}^{\prime}\left(\omega\left(g_{b}(p)\right)-\log \log x\right)^{2} \ll \pi(x) \log \log x .
$$

This completes the proof of Theorem 1 . 


\section{An Erdös-Kac analogue of $\omega\left(g_{b}(p)\right)$}

In this section, we give a proof of Theorem 3. More precisely, under the GRH we prove that there exists a normal distribution for the quantity

$$
\frac{\omega\left(g_{b}(p)\right)-\log \log p}{\sqrt{\log \log p}} .
$$

Our proof is dependent on the following theorem.

Theorem 10 (Liu [15]). Let $E / \mathbb{Q}$ be an elliptic curve. We have (assuming the GRH if $E$ is non-CM)

$$
\begin{aligned}
& \lim _{x \rightarrow \infty} \frac{1}{\pi(x)} \#\left\{p \leq x \mid p \text { is of good reduction and } \frac{\omega\left(\# \bar{E}\left(\mathbb{F}_{p}\right)\right)-\log \log p}{\sqrt{\log \log p}} \leq \gamma\right\} \\
& =G(\gamma) .
\end{aligned}
$$

Proof of Theorem 3. As in the proof of Theorem 1, we have

$$
\begin{aligned}
\frac{\omega\left(\# \bar{E}\left(\mathbb{F}_{p}\right)\right)-\log \log p}{\sqrt{\log \log p}} & \geq \frac{\omega\left(g_{b}(p)\right)-\log \log p}{\sqrt{\log \log p}} \\
& \geq \frac{\omega\left(\# \bar{E}\left(\mathbb{F}_{p}\right)\right)-\log \log p}{\sqrt{\log \log p}}-\frac{\omega\left(i_{b}(p)\right)}{\sqrt{\log \log p}} .
\end{aligned}
$$

For any $\varepsilon>0$ and $\alpha, \beta \in \mathbb{R}$ with $\alpha<\beta$, define the set

$$
S(\varepsilon, \alpha, \beta)=\left\{p \mid p \text { is of good reduction, } \alpha<p \leq \beta \text {, and } \frac{\omega\left(i_{b}(p)\right)}{\sqrt{\log \log p}} \geq \varepsilon\right\} .
$$

Let $N(\varepsilon, \alpha, \beta)$ be the cardinality of $S(\varepsilon, \alpha, \beta)$. We have

$$
N(\varepsilon, 0, x) \leq \pi(\sqrt{x})+N(\varepsilon, \sqrt{x}, x) .
$$

Notice that

$$
\sum_{p \leq x}^{\prime} \omega\left(i_{b}(p)\right) \geq \sum_{p \in S(\varepsilon, \sqrt{x}, x)} \omega\left(i_{b}(p)\right) \geq N(\varepsilon, \sqrt{x}, x) \cdot \varepsilon \sqrt{\log \log x-\log 2} .
$$

Since $\omega^{2}\left(i_{b}(p)\right) \geq \omega\left(i_{b}(p)\right)$, Lemma 8 implies that

$$
N(\varepsilon, \sqrt{x}, x) \ll \frac{\pi(x)}{\sqrt{\log \log x}}=o(\pi(x)) .
$$

It follows that

$$
N(\varepsilon, 0, x)=o(\pi(x))
$$


Vol. 80 (2005) A prime analogue of the Erdös-Pomerance conjecture for elliptic curves 767

Thus for $\gamma \in \mathbb{R}$ we obtain

$$
\begin{aligned}
& \#\left\{p \leq x \mid p \text { is of good reduction and } \frac{\omega\left(g_{b}(p)\right)-\log \log p}{\sqrt{\log \log p}} \leq \gamma\right\} \\
& \leq \#\{p \leq x \mid p \text { is of good reduction and } \\
& \left.\qquad \frac{\omega\left(\# \bar{E}\left(\mathbb{F}_{p}\right)\right)-\log \log p}{\sqrt{\log \log p}}-\frac{\omega\left(i_{b}(p)\right)}{\sqrt{\log \log p}} \leq \gamma\right\} \\
& \leq \#\{p \leq x \mid p \text { is of good reduction and } \\
& \left.\frac{\omega\left(\# \bar{E}\left(\mathbb{F}_{p}\right)\right)-\log \log p}{\sqrt{\log \log p}} \leq \gamma+\varepsilon\right\}+o(\pi(x)) .
\end{aligned}
$$

Also we have

$$
\begin{aligned}
\#\left\{p \leq x \mid p \text { is of good reduction and } \frac{\omega\left(g_{b}(p)\right)-\log \log p}{\sqrt{\log \log p}} \leq \gamma\right\} \\
\quad \geq \#\left\{p \leq x \mid p \text { is of good reduction and } \frac{\omega\left(\# \bar{E}\left(\mathbb{F}_{p}\right)\right)-\log \log p}{\sqrt{\log \log p}} \leq \gamma\right\} .
\end{aligned}
$$

Combine all of the above results with Theorem 10. As $x \rightarrow \infty$, for all $\varepsilon>0$ we obtain

$$
\begin{aligned}
& G(\gamma) \leq \lim _{x \rightarrow \infty} \frac{1}{\pi(x)} \#\{p \leq x \mid p \text { is of good reduction and } \\
& \left.\qquad \frac{\omega\left(g_{b}(p)\right)-\log \log p}{\sqrt{\log \log p}} \leq \gamma\right\} \leq G(\gamma+\varepsilon) .
\end{aligned}
$$

Since $G(\gamma)$ is a continuous function, for any $\varepsilon>0$ we have

$$
G(\gamma+\varepsilon)=G(\gamma)+O(\varepsilon) .
$$

Let $\varepsilon \rightarrow 0$. It follows that under the GRH,

$$
\begin{aligned}
\lim _{x \rightarrow \infty} & \frac{1}{\pi(x)} \#\left\{p \leq x \mid p \text { is of good reduction and } \frac{\omega\left(g_{b}(p)\right)-\log \log p}{\sqrt{\log \log p}} \leq \gamma\right\} \\
& =G(\gamma) .
\end{aligned}
$$

This completes the proof of Theorem 3.

\section{References}

[1] M. I. Bachmakov, Un théorème de finitude sur la cohomologie des courbes elliptiques. C. R. Acad. Sci. Paris Sér. A-B 270 (1970), A999-A1101. Zbl 0194.52303 MR 0269653 
[2] J. Coates and A. Wiles, On the conjecture of Birch and Swinnerton-Dyer. Invent. Math. 39 (1977), 223-251. Zbl 0359.14009 MR 0463176

[3] P. D. T. A. Elliott, Probabilistic number theory. Vol. I and II, Grundlehren Math. Wiss. 239, 240, Springer-Verlag, New York, Berlin 1979. Zbl 0431.10029 MR 0551361 Zbl 0431.10030 MR 0560507

[4] P. Erdös, On the normal order of prime factors of $p-1$ and some related problems concerning Euler's $\phi$-functions. Quart. J. Math. (Oxford) 6 (1935), 205-213. Zbl 0012.14905

[5] P. Erdös and M. Kac, The Gaussian law of errors in the theory of additive number theoretic functions. Amer. J. Math. 62 (1940), 738-742. JFM 66.0172.02 MR 0002374

[6] P. Erdös and C. Pomerance, On the normal number of prime factors of $\varphi(n)$. Rocky Mountain J. Math. 15 (1985), 343-352. Zbl 0617.10037 MR 0823246

[7] R. Gupta and M. R. Murty, Primitive points on elliptic curves. Compositio Math. 58 (1986), 13-44. Zbl 0598.14018 MR 0834046

[8] G. H. Hardy and S. Ramanujan, The normal number of prime factors of a number $n$. Quart. J. Pure. Appl. Math. 48 (1917), 76-97. JFM 46.0262.03

[9] C. B. Haselgrove, Some theorems in the analytic theory of numbers, J. London Math. Soc. 26 (1951), 273-277. Zbl 0043.04704 MR 0044564

[10] J. Kubilius, Probabilistic methods in the theory of numbers. Transl. Math. Monogr. 11, Amer. Math. Soc., Providence, R.I., 1964. Zbl 0133.30203 MR 0160745

[11] J. Lagarias and A. Odlyzko, Effective versions of the Chebotarev density theorem. In Algebraic number fields (A. Fröhlich, ed.), Academic Press, New York 1977, 409-464. Zbl 0362.12011 MR 0447191

[12] S. Lang and H. Trotter, Primitive points on elliptic curves. Bull. Amer. Math. Soc. 83 (1977), 289-292. Zbl 0345.12008 MR 0427273

[13] S. Li and C. Pomerance, On generalizing Artin's conjecture on primitive roots to composite moduli. J. Reine Angew. Math. 556 (330), 205-224. Zbl 1022.11049 MR 1971146

[14] Y.-R. Liu, Prime divisors of number of rational points on elliptic curves with complex multiplication. To appear in Bull. London Math. Soc.

[15] Y.-R. Liu, Generalizations of the Turán and the Erdös-Kac theorems. Ph.D. thesis, Harvard 2003.

[16] S. A. Miri and V. K. Murty, An application of sieve methods to elliptic curves. In Progress in Cryptology -INDOCRYPT, Lecture Notes in Comput. Sci. 2247, Springer-Verlag, Berlin 2001, 91-98. Zbl 1011.94543 MR 1934487

[17] M. R. Murty and F. Saidak, Non-abelian generalizations of the Erdös-Kac theorem. Canad. J. Math. 56 (2004), 356-372. Zbl 1061.11052 MR 2040920

[18] J.-P. Serre, Propriétés galoisiennes des points d'ordre fini des courbes elliptiques. Invent. Math. 15 (1972), 259-331. Zbl 0235.14012 MR 0387283

[19] J.-P. Serre, Quelques applications du théorème de densité de Chebotarev, Inst. Hautes Études Sci. Publ. Math. 54 (1981), 123-201. Zbl 0496.12011 MR 0644559

[20] H. Shapiro, Distribution functions of additive arithmetic functions. Proc. Nat. Acad. Sci. U.S.A. 42 (1956), 426-430. Zbl 0071.04202 MR 0079609 
Vol. 80 (2005) A prime analogue of the Erdös-Pomerance conjecture for elliptic curves 769

[21] J. H. Silverman, The arithmetic of elliptic curves. Grad. Texts in Math. 106, SpringerVerlag, New York 1986. Zbl 0585.14026 MR 1329092

[22] J. H. Silverman, Advanced topics in the arithmetic of elliptic curves. Grad. Texts in Math. 151, Springer-Verlag, New York 1994. Zbl 0911.14015 MR 1312368

[23] P. Turán, On a theorem of Hardy and Ramanujan. J. London Math. Soc. 9 (1934), 274-276. Zbl 0010.10401

Received March 23, 2004

Yu-Ru Liu, Department of Pure Mathematics, University of Waterloo, Waterloo, Ontario, Canada N2L 3G1

E-mail: yrliu@math.uwaterloo.ca 